\title{
Multi-Stage Pyocyanic Spondylodiscitis: A Case Report
}

\section{Ilyas El Kassimi*, Adil Rkiouak, Salah-Eddine Hammi, Nawal Sahel, Oumama Jamal, Youssef Sekkach}

Department of Internal Medicine, Mohammed V Military Teaching Hospital, Mohammed V university Souissi, Rabat, Morocco

*Corresponding Author: Ilyas El Kassimi, Department of Internal Medicine, Mohammed V Military Teaching Hospital, Mohammed V university Souissi, Rabat, Morocco, Tel : +212614742828; E-mail: dr.elkassimiilyas@gmail.com

Received: 12 April 2018; Accepted: 22 April 2018; Published: 26 April 2018

\begin{abstract}
Spondylodiscitis is an intraspinal infection that affects the intervertebral disk and adjacent vertebral bodies. Neurological signs represent urgency and a criterion of gravity. Magnetic resonance imaging (MRI) is the baseline assessment to make the diagnosis. Pyocyanic bacillus represents less than $5 \%$ of bacterial causes. We report the case of a 79-year-old woman who progressively presented paresthesia of the four limbs with flaccid tetraparesis. MRI showed multi-stage cervico-dorsal involvement. Based on that, computed tomography-guided disco vertebral biopsy was performed and permitted to isolate a pyocyanic agent. The treatment consisted of a double antibiotherapy with resuscitation measures. The evolution was favorable, and the entry gate remained mysterious.
\end{abstract}

Keywords: Spondylodiscitis; Pyocyanic; Tetraparesis; Diagnosis; Treatment

\section{Introduction}

Spondylodiscitis is an infection of the disc and adjacent vertebrae. The form due to the pyocyanic bacillus remains rare but serious, and the multistage localization exposes to the risk of spinal compression.

\section{Case presentation}

A 79-year-old female patient, with a history of breast cancer treated 8 years ago by lumpectomy with lymph node dissection followed by a chemoradiotherapy with good evolution. She was admitted for tetraparesis, which has been present during the last three months, paresthesia of both upper limbs worsening in a progressive manner evolving 
towards a total motor deficit of the four limbs, with colonic abdominal pain and constipation, all evolving in a context of apyrexia and deterioration of general condition. There was no antecedent of tuberculosis or concept of tuberculous contagion, nor recent spinal procedure.

The clinical examination found, an obese patient with a body mass index at $35 \mathrm{~kg} / \mathrm{m}^{2}$, a flaccid tetraparesis without individualizable sensory level, the palpation of the spinous and para-spinous processes did not produce pain. Cardiovascular examination did not find a heart murmure, examination of the breasts and gonglion areas was normal. The abdomen was soft, compressible, painless and had no hepatosplenomegaly.

The morphological assessment including a spinal MRI showed multiple localizations with C6-C7 and D1-D2 involvement with medullary compression at these levels (Figure 1), a starting lesion at D5-D6 and ivory vertebra aspect of D8 (Figure 2). Biological assessment showed an inflammatory syndrome with erythrocyte sedimentation rate at $105 \mathrm{~mm}$ at the $1 \mathrm{st}$ hour, and C-reactive protein at $81 \mathrm{mg} / \mathrm{l}$, a moderate leukocytosis at $11800 / \mathrm{mm}^{3}$. Bacteriological exam of a computed tomography-guided disco vertebral biopsy showed a paucycellular sample at the first analysis and the culture confirmed the presence of a Pyocyanic. Histopathological study did not show granuloma or tumor cells. The bacterial entry gate was undefined: blood cultures, cytobacterioligical urine exam, transthoracic echocardiogram and chest X-ray were normal.

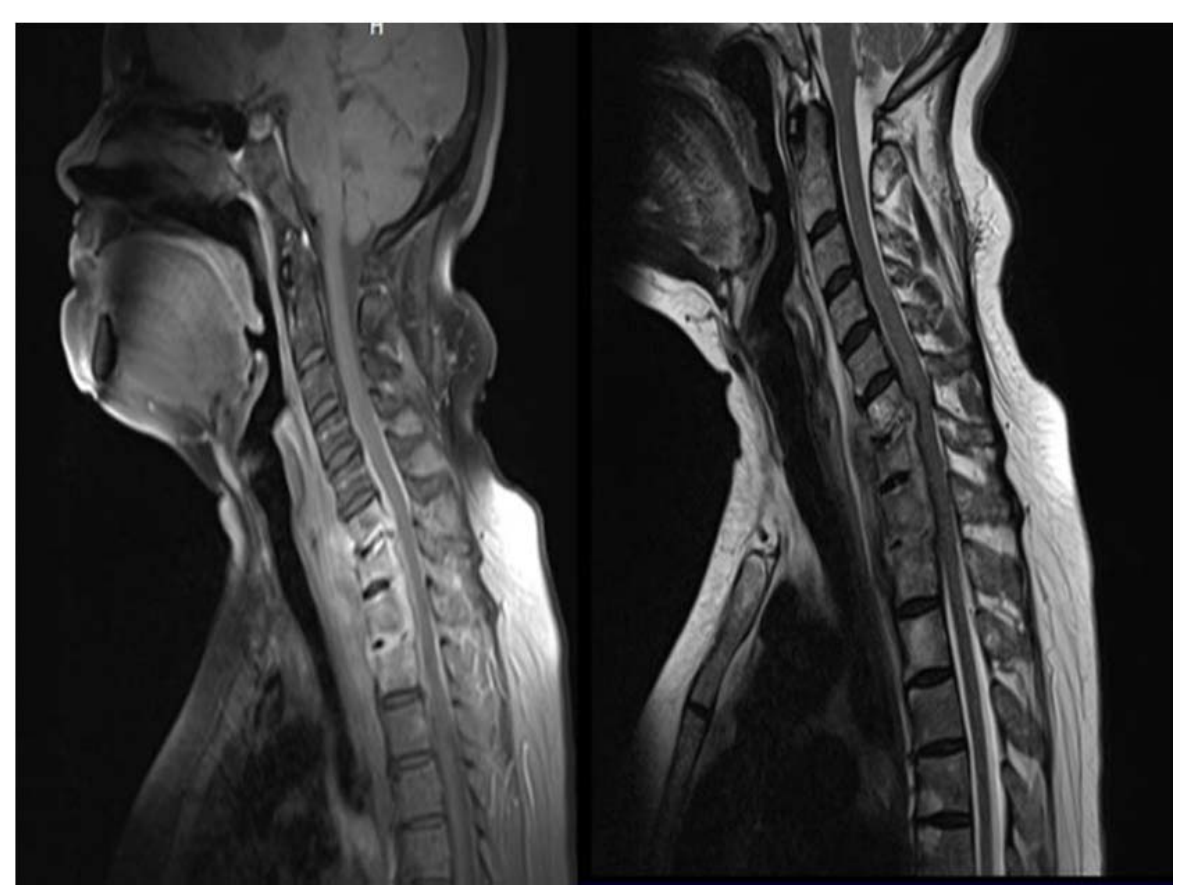

Figure 1: MRI showing C6-C7 and D1-D2 involvement with medullary compression at these levels. 


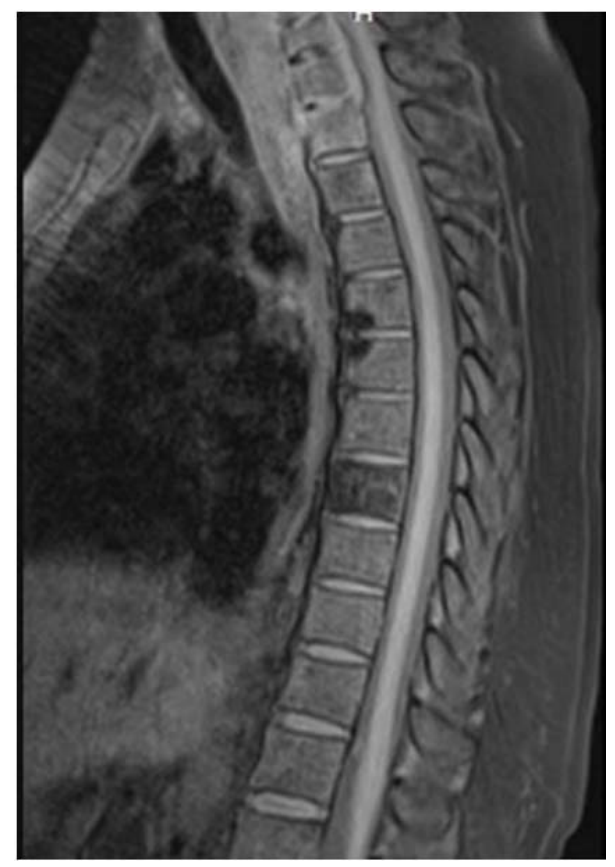

Figure 2: MRI showing D5-D6 involvement and D8 ivory vertebra aspect

Treatment included intravenous antibiotics: third-generation cephalosporin (ceftazidim) combined with fluoroquinolone (Ciprofloxacin), WHO stage 2 analgesic, prophylactic anticoagulation, gastric protection, anti bed sores mattress, transit regulator with a neck brace and physiotherapy sessions. According to neurosurgeons, there was no indication of surgery. The evolution was favorable, with decrease of inflammatory parameters and slight recovery of the clinical symptomatology.

\section{Discussion}

Spondylodiscitis is an intraspinal infection that affects the intervertebral disk and adjacent vertebral bodies. It is rare but serious: 4 to $7 \%$ of osteomyelitis, more common in men (sex ratio 3/2), with a bimodal frequency peak: in adolescence and over 50 years. The typical clinical symptomatology is characterized by inflammatory (in> 50\%) pain (90\%), the fever is inconstant, and the neurological signs represent the urgency and a sign of gravity as in our case.

The diagnostic assessment includes morphological investigations. Spinal X-ray shows in a delayed way a fuzzy and erased aspect of the cortical border of a vertebral plateau, erosions of the anterior corner of the vertebral plateau, a disc pinch, a spindle swelling of soft tissue, CT can show a decrease in the density of the disc, erosions and geodes of the discs. However, MRI remains the examination of choice for the early diagnosis especially in the presence of neurological symptomatology, it shows T2 hypersignal disc, T1 hyposignal and T2 hypersignal of both vertebrae $[1,2]$. 
Biological assays objectify nonspecific inflammatory syndrome with neutrophilic hyperleukocytosis. Bacteriological diagnosis is made either by using a computed tomography-guided disco vertebral biopsy or by peripheral samples including blood cultures and presumed entry gates. The most common pathogens are Staphylococcus aureus (2050\%), followed by streptococci, coagulase-negative staphylococci, enterobacteria (especially Escherichia coli), Pseudomonas aeruginosa $<5 \%$ - as in our case - and enterococci.

The localizations of spinal lesions found in order of frequency are the lumbar spine (more than $50 \%$ of the cases), then dorsal spine (30\%) and cervical spine (20\%). This last localization - as in our case - represents an element of gravity because of the frequency of spinal cord injuries at this level.

Antibiotic treatment recommended by the French Language Society of Infectious Pathology for Pseudomonas spondylodiscitis is as follow: in the initial phase, ticarcillin or piperacillin-tazobactam combination or ceftazidim, associated with ciprofloxacin or a venous aminoglycoside. As maintenance treatment, ciprofloxacin can be used orally as monotherapy after initial two-week parenteral therapy, subject to the susceptibility of the strain [3]. Monitoring and evaluation should be based on clinical, inflammatory status and radiology.

\section{Conclusion}

Spinal infection still warrants rapid, sometimes urgent, diagnostic and therapeutic management based on clinical practice, mainly MRI and bacteriological exams.

\section{References}

1. N Sans, M Faruch, F Lapègue, A Ponsot, H Chiavassa, JJ Railhac. Infection du rachis - Spondylodiscites. Journal de Radiologie Diagnostique et Interventionnelle 93 (1993): 551-560.

2. A Moraux, E Kermarrec, E Czarnecki, N Boutry, X. Demondion et A Cotten. Infections rachidiennes : aspects typiques et atypiques. J Radiol 91 (2010): 1049-1056.

3. Chidiac, Christian, et al. Recommandations pour la pratique clinique Spondylodiscites infectieuses primitives, et secondaires à un geste intradiscal, sans mise en place de matériel." Médecine et maladies infectieuses 37 (2007): 554-572.

Citation: Ilyas El Kassimi, Adil Rkiouak, Salah-Eddine Hammi, Nawal Sahel, Oumama Jamal, Youssef Sekkach. Multi-Stage Pyocyanic Spondylodiscitis: A Case Report. Archives of Clinical and Biomedical Research 2 (2018): 113-116.

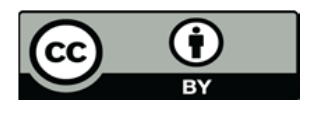

This article is an open access article distributed under the terms and conditions of the Creative Commons Attribution (CC-BY) license 4.0 